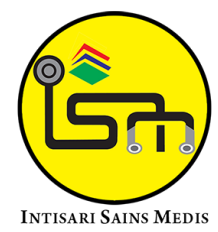

Published by Intisari Sains Medis

\title{
Effectivity of glutathione and curcumin in hepatic fibrosis induced by cholestasis in Wistar rats
}

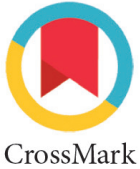

Rodijaya Putra ${ }^{1 *}$, Adeodatus Yuda Handaya ${ }^{2}$, Agus Barmawi ${ }^{2}$

'Student of Digestive Surgery Subspecialist Program, Faculty of Medicine, Universitas Gadjah Mada, Yogyakarta, Indonesia;

${ }^{2}$ Department of Digestive Surgery. Faculty of Medicine, Universitas Gadjah Mada, Yogyakarta, Indonesia.

*Corresponding author:

Rodijaya Putra;

Student of Digestive Surgery Subspecialist Program, Faculty of Medicine, Universitas Gadjah Mada, Yogyakarta, Indonesia;

rodijayaputra@yahoo.com

Received: 2021-03-10

Accepted: 2021-06-30

Published: 2021-07-15

\section{ABSTRACT}

Background: Cholestasis occurs from the accumulation of bile product in the biliary tract due to obstruction of the hepatobiliary tract. Without early interventions, this condition will be continuing as chronic hepatic injury and form hepatic fibrosis, ending in cirrhosis. Glutathione (GSH) and curcumin were known as antioxidants and anti-inflammatory used as a therapeutic agent for the treatment of hepatopathy. This study aims to determine the effectivity of GSH and curcumin in the hepatic fibrosis mechanism induced by cholestasis.

Methods: This is an experimental study using Wistar rats. They are divided into three groups of 5 rats each. All the rats regard the choledochus duct ligation surgery, rats without intervention define as first group (control), other rats treated with GSH as the second group and the third group consist of rats treated with curcumin. Data were analyzed using SPSS version 17 for Windows.

Results: There was liver injury and hepatic fibrosis induced by cholestasis from this study. This condition was significantly lower in groups treated with GSH and curcumin $(p<0.05)$. Therefore, there was a different result between GSH and curcumin in reducing the process of liver injury and hepatic fibrosis, but the difference was not statistically significant $(p>0.05)$. Conclusion: GSH and curcumin were statistically effective in reducing liver injury and hepatic fibrosis induced by cholestasis. Despite, there were no significant difference results between them in reducing liver injury and hepatic fibrosis.

Keywords: Hepatic fibrosis, Glutathione, Curcumin, Cholestasis.

Cite This Article: Putra, R., Handaya, A.Y., Barmawi, A. 2021. Effectivity of glutathione and curcumin in hepatic fibrosis induced by cholestasis in Wistar rats. Intisari Sains Medis 12(2): 459-462. D0I: 10.15562/ism.v12i2.997

\section{INTRODUCTION}

Cholestasis occurs from obstruction of bile product in the biliary tract due to obstruction of the intrahepatic and extrahepatic hepatobiliary tract. ${ }^{1}$ The continuum of cholestasis is liver injury and structurally abnormal liver architecture defined by hepatic fibrosis and ending in cirrhosis. The other causes of liver injury and hepatic fibrosis are viral infection, metabolic, ethanol abused and malignancy. ${ }^{1}$ American's Society of Liver disease accounts for approximately 15\% of causes of liver failure is cholestasis. Europe Association of Liver Cirrhosis confirmed $20 \%$ of hepatic fibrosis is due to abnormality of the biliary tract that induced cholestasis. ${ }^{2}$ There is no available data that shows the percentage of hepatic fibrosis generated by cholestasis in Indonesia. Numerous cholestasis causes include infection, bile duct stone, congenital and acquired anatomical abnormalities, and malignancy. ${ }^{1,2}$

Sometimes, patients come to the doctor in late cholestasis conditions. Therefore, in that condition, liver injury and focal or extended hepatic fibrosis should be suspected. It is a dilemma for the clinician to treat this condition. Management of hepatic fibrosis induced by cholestasis should be as soon as possible, consists of surgical drainage or medicamentosa. Currently, there were several medications available for hepatic fibrosis, such as Ursodeoxycholic acid (UDCA), Curcumin, Vitamins (C and E) and antioxidants agents, to assist hepatocyte healing. ${ }^{3}$ However, those drugs work in unknown mechanisms in reducing the injury and hepatic fibrosis induced by cholestasis. ${ }^{4}$

Glutathione (GSH) is a protein that consists of three primary amino acids such as L-Glutamic Acid, L-Cysteine, and L-Glycine. GSH is the main thiol in cells act as a cofactor in several antioxidants and detoxifying enzymes. GSH is the mother of antioxidants in mammals. The liver produces a significant amount of GSH and excretes it into plasma and bile. ${ }^{5}$ Cholestasis is reducing the availability of GSH in mice blood. ${ }^{6}$ This condition is reducing the ability of the antioxidant system in mice. In one experimental study, GSH treatment $300 \mathrm{mg} / \mathrm{kg}$ oral for 7 days reduced total bilirubin, ALT, AST, and proinflammatory cytokine levels in the systemic circulation. GSH also impaired the activity of free radicals Nitrotyrosine (NT), Nitric Oxide (NO) and Malondialdehyde (MDA). ${ }^{7} \mathrm{GSH}$ increase responsivity of endothelium that impair the activity of hepatic stellate cell and prevent hepatic fibrosis. GSH impairs Tumor Necrotizing Factor- $\alpha$ (TNF- $\alpha$ ) and Interleukin-1 $\beta$ (IL-1 $\beta$ ), the proinflammatory cytokines that promote the hepatic fibrosis process.

Curcumin is an active polyphenol 
extracted from Curcuma xanthorriza rhizome. Curcumin has known as an antioxidant, anti-inflammatory and anti-tumor in chronic hepatic disease and malignancy. ${ }^{8}$ The proinflammatory cytokines increase in cholestasis. These cytokines induce Janus Kinase 2 (JAK-2) and activate the inflammatory cascade in which increases Reactive Oxygen Species (ROS), Hypoxia Inducible Factor (HIF-1) and Transforming Growth Factor (TGF 1 $\beta$ ). All of these cytokines promote hepatic fibrosis. ${ }^{9}$ Curcumin impairs the proinflammatory cytokines. Therefore, it was used as an agent therapeutic of hepatic fibrosis induced by cholestasis. ${ }^{10}$

The diagnosis of injury and hepatic fibrosis is performed by invasive and non-invasive examinations. Imaging examinations such as Magnetic Resonance Elastography (MRE) is a non-invasive technique, expensive, and limited. Ultrasonography with Transient elastography also has a limitation in access. Laboratory examinations such as Aminotransferase to platelet ratio index (APRI) or Fibrosis-4 (FIB-4) are standard tests despite a low accuracy level. A laboratory examination using index or scoring such as Forns Index, Fibro Index, and the BMI, Age, ALT, and Triglyceride (BAAT) score also does not reveal good accuracy. A gold standard examination of hepatic fibrosis is histopathological evaluation. $^{1}$ It shows the appearance of liver injury characterized by the accumulation of inflammatory cells, steatosis, necrotic combined with fibrotic and collagen confined to a sophisticated finding of hepatic fibrosis. ${ }^{11}$ Therefore, this study aims to determine the effectiveness of two kinds of drugs, Glutathione and Curcumin, in hepatic fibrosis induced by cholestasis in Wistar rats.

\section{METHODS}

The experimental animals used in this study were adult (2-3 months) male Wistar rats (weighing 200-250 gram). These rats were appropriate for this research because the pathophysiology of disease and anatomy of intraperitoneal organs tend to be similar to humans based on the previous study. ${ }^{12}$ The rat's choledochal duct remains an inappropriate size that is

Table 1. Evaluation of scoring system by Kruskal-Wallis

\begin{tabular}{lccc}
\multicolumn{1}{c}{ Group } & $\mathbf{n}$ & Mean+SD & p \\
\hline Evaluation with A scoring system & & & \\
$\quad$ Control & 5 & $2.46 \pm 0.38$ & $0.005^{*}$ \\
Glutathione & 5 & $0.66 \pm 0.34$ & \\
$\quad$ Curcumin & 5 & $1.06 \pm 0.37$ & \\
Evaluation with B scoring system & & & \\
$\quad$ Control & 5 & $4.00 \pm 0.00$ & $0.005^{\star}$ \\
$\quad$ Glutathione & 5 & $1.53 \pm 0.30$ & \\
$\quad$ Curcumin & 5 & $2.33 \pm 0.33$ & \\
\hline
\end{tabular}

helpful to identify in surgical procedure and reduce the bias and failure occur.

All rats were divided into three groups of 5 rats each. The number of samples determined with the Federer formula in which the total sample is 15 rats. Eligible samples that met inclusion criteria performed a laparotomy surgical with double ligation with 3.0 non-absorbable thread (Silk) and resection of choledochal duct (Common Bile Duct/CBD). After that, all samples were prepared with no intervention at all in 3 weeks. After 3 weeks, each group-administered different intervention. The first group as control was not performed anything at all. The second group was administered with $3.9 \mathrm{mg} / \mathrm{kg}$ oral Glutathione each day for 2 weeks. The third group was administered with $1.3 \mathrm{mg} /$ $\mathrm{kg}$ oral Curcumin each day for 2 weeks.

Two weeks later, all rats of three groups performed a termination. After that, the liver organ of all experimental animals was obtained by resection. The resected liver has enrolled a fixation in formalin buffer $10 \%$ following histopathological examination using Hematoxylin Eosin (HE). This staining aims to determine the process of liver injury and the extension of hepatic fibrosis from each sample of the group. This follows with statistical analysis to evaluate the effectiveness of GSH and Curcumin in liver injury and hepatic fibrosis.

Data obtained were analyzed with Shapiro-Wilk for the normality test. In addition, a non-parametric statistical analysis Kruskal-Wallis was performed to test the value of the mean difference in the three groups tested. The p-value below 0.05 was considered statistically significant. Data were analyzed using SPSS version 17 for Windows.

\section{RESULTS}

Regarding Kruskal-Wallis analysis, it was obtained a mean value that statistically significant in evaluating fibrous tissue/ collagen with A scoring system and cytology with B scoring system in each treated group $(\mathrm{p}<0.05)$ (Table 1). Glutathione treated group revealed the lowest mean value $(0.66 \pm 0.34)$ compared to the curcumin-treated group $(1.06 \pm 0.37)$ and control $(2.46 \pm 0.38)$ using A scoring system (Table 1). On the other hand, using the B scoring system obtained the lowest mean value in the glutathione treated group $(1.53 \pm 0.30)$ compared to the curcumin-treated group $(2.33 \pm 0.33)$ and control $(4.00 \pm 0.00)$. The analysis between the control group and both glutathione and curcumin-treated group showed statistically significant differences $(p<0.05)$. The analysis between the glutathione treated and curcumintreated groups using the A or B scoring system showed differences, but it was not statistically significant ( $\mathrm{p}>0.05)$ (Table 1$)$.

\section{DISCUSSION}

Cholestasis increased the availability of free radicals (Nitrotyrosine, Nitric Oxide, and Malondyaldehyde) and pro-inflammatory cytokines (Tumor Necrotizing Factor and Interleukin 1- $\beta$ ). Free radicals and pro-inflammatory cytokines induced Hepatic Stellate Cell (HSC) and recruited Extracellular Matrix (ECM).${ }^{13}$ Inflammatory cell accumulation, steatosis and necrotic liver cells were a continuum process to liver injury. The livers' regenerating system would repair this injury; unless it was failed, this process ended with hepatic fibrosis. ${ }^{5,7}$ In this study, all experimental rats have performed a 
CBD double ligation with 3.0 Silk and resection to prevent the $\mathrm{CBD}$ regeneration process. $^{14}$ Therefore, the CBD was occluded and induced cholestasis process.

Regarding previous study findings, cholestasis induced by ligation of CBD for more than 3 weeks in mice was enough to cause hepatic fibrosis. ${ }^{15}$ This condition occurred due to the accumulation of bile products and bile acid-induced biliary cells activating inflammatory mediators such as TNF- $\alpha$, Endothelin-1, PDGF and others. In addition, they promoted HSC and recruited ECM and collagen. Those complex process was related to pro-inflammatory factors and free radical agents. ${ }^{16} \mathrm{~A}$ liver injury process that was not appropriately treated would be ended with fibrosis and cirrhosis. ${ }^{17}$ The management of cholestasis was surgical biliary drainage. It would prevent the following injury and fibrosis.

On the other hand, sometimes the drainage could not continually be enrolled to people who suffered cholestasis. It might be due to the patient's condition, lack of medical utility and lack of medical staff's competency. It was also not an easy decision to perform a surgical way nowadays, due to pandemic Covid 19. Therefore, to prevent the neglected case of cholestasis, we should consider the other significant interventions to do as soon as possible and avoid fibrosis and cirrhosis.

GSH and Curcumin were drugs acting as antioxidants, disrupting free radicals and pro-inflammatory factors to work and preventing liver injury and hepatic fibrosis. Despite their antioxidant effect, literature informed that both of them had a different mechanism of action. ${ }^{18}$ Regarding this condition, we would compare their effectiveness and other effects in liver injury and hepatic fibrosis. GSH was a mother of antioxidants and usually produced by the liver in specific amounts as a free radical controller. In mammals, these natural antioxidants would be decreasing, in concordance with impairing of biliary cells. ${ }^{18}$ Thus, we should add external antioxidants to enhance their protecting activity in liver cells. Curcumin was an active polyphenol. It had antioxidants and mild anti-inflammation. Curcumin was not produced endogenously. It acted through the kinase pathway and prevented the continuum of liver injury and hepatic fibrosis. ${ }^{18}$ GSH and Curcumin were given for 2 weeks based on daily dose. The other studies recommended GSH and Curcumin were given for 10-14 days to reach the maximal effect. Eshaghian A et al. recommended Curcumin with a dose of $100 \mathrm{mg} / \mathrm{kg}$ each day for 10 days. $^{8}$

This study revealed that liver injury and hepatic fibrosis were reduced significantly. This was proper to previous literature and studies. Thus, this study could be another reference of non-surgical interventions in liver injury and hepatic fibrosis-induced cholestasis. ${ }^{19}$ GSH and Curcumin should be considered to give before the surgery was performed. Based on statistical analysis findings, the effect of GSH and Curcumin to reduce liver injury and hepatic fibrosis did not differ significantly. Still, the GSH score is better than Curcumin one. This study showed that, even though the mechanism of action GSH and Curcumin differ, their effect was fair but better than the control group. It may happen due to their similar activity in decreasing the inflammatory process and free radicals involved in hepatic fibrosis.

The histopathology examination using HE staining of three groups showed a sophisticated liver injury process and hepatic fibrosis induced by cholestasis. ${ }^{19,20}$ The accumulation of bile promotes inflammation of biliary cells and induces inflammatory cells, steatosis and necrotizing cells. The hallmark of hepatic fibrosis in histopathology examination was the accumulation of fibrous tissue or collagen at all specimens in different quantities. ${ }^{20,21}$ The different amounts of fibrous tissue/collagen findings resulted from our interventions (GSH and Curcumin). Thus, these findings concluded that both of them significantly reduced liver injury and hepatic fibrosis.

\section{CONCLUSION}

Regarding the results of this study, GSH and Curcumin were effective and statistically significant in reducing the injury process and hepatic fibrosis induced by cholestasis. However, despite their effectiveness, the differential effect between GSH and Curcumin was not statistically significant.

\section{CONFLICT OF INTEREST}

There is no competing interest regarding the manuscript.

\section{ETHICS CONSIDERATION}

Ethics approval has been obtained from the Ethics Committee, Faculty of Medicine, Universitas Gadjah Mada, Yogyakarta, Indonesia, prior to the study being conducted.

\section{FUNDING}

None.

\section{AUTHORS CONTRIBUTION}

All authors equally contribute to the study from the conceptual framework, data gathering, data analysis until reporting the study results through publication.

\section{REFERENCES}

1. Bataller R, Brenner DA. Liver fibrosis. J Clin Invest. 2005;115(2):209-218.

2. Mormone E, George J, Nieto N. Molecular pathogenesis of hepatic fibrosis and current therapeutic approaches. Chem Biol Interact. 2011;193(3):225-231.

3. Friedman SL. Liver fibrosis -- from bench to bedside. J Hepatol. 2003;38 Suppl 1:S38-S53.

4. Cederbaum AI. Hepatoprotective effects of S-adenosyl-L-methionine against alcoholand cytochrome P450 2E1-induced liver injury. World J Gastroenterol. 2010;16(11):13661376.

5. Hirota M, Sugi K, Inoue M. Dynamic aspects of glutathione metabolism in obstructive jaundice. J Gastroenterol. 1994;29(5):588-592.

6. Panozzo MP, Basso D, Balint L, Biasin MR, Bonvicini P, Metus $\mathrm{P}$, et al. Altered lipid peroxidation/glutathione ratio in experimental extrahepatic cholestasis. Clin Exp Pharmacol Physiol. 1995;22(4):266-271.

7. Chen J, Wu F, Long Y, Yu W. Glutathione Supplementation Attenuates Oxidative Stress and Improves Vascular Hyporesponsiveness in Experimental Obstructive Jaundice. Oxid Med Cell Longev. 2015;2015:486148.

8. Eshaghian A, Khodarahmi A, Safari F, Binesh F, Moradi A. Curcumin attenuates hepatic fibrosis and insulin resistance induced by bile duct ligation in rats. Br J Nutr. 2018;120(4):393-403.

9. El Swefy S, Hasan RA, Ibrahim A, Mahmoud MF. Curcumin and hemopressin treatment attenuates cholestasis-induced liver fibrosis in rats: role of $\mathrm{CB} 1$ receptors. Naunyn Schmiedebergs Arch Pharmacol. 2016;389(1):103-116.

10. Ghoreshi ZA, Kabirifar R, Safari F, Karimollah A, Moradi A, Eskandari-Nasab E. Hepatoprotective effects of curcumin in rats 
after bile duct ligation via downregulation of Rac1 and NOX1. Nutrition. 2017;36:72-78.

11. Liedtke C, Luedde T, Sauerbruch T, Scholten D, Streetz K, Tacke F, et al. Experimental liver fibrosis research: update on animal models, legal issues and translational aspects. Fibrogenesis Tissue Repair. 2013;6(1):1-24.

12. Turk O, Badak B, Ates E, Dundar E, Sutken E. The role of growth factors on hepatic damage in rats with obstructive jaundice. Springerplus. 2016;5(1):1274.

13. Li S, Tan HY, Wang N, et al. The Role of Oxidative Stress and Antioxidants in Liver Diseases. Int J Mol Sci. 2015;16(11):2608726124.

14. Cabrera-Rubio R, Patterson AM, Cotter PD, Beraza N. Cholestasis induced by bile duct ligation promotes changes in the intestinal microbiome in mice. Sci Rep. 2019;9(1):12324.
15. Tag CG, Sauer-Lehnen S, Weiskirchen S, et al. Bile duct ligation in mice: induction of inflammatory liver injury and fibrosis by obstructive cholestasis. J Vis Exp. 2015;(96):52438.

16. Wang ME, Chen YC, Chen IS, Hsieh SC, Chen SS, Chiu CH. Curcumin protects against thioacetamide-induced hepatic fibrosis by attenuating the inflammatory response and inducing apoptosis of damaged hepatocytes. J Nutr Biochem. 2012;23(10):1352-1366.

17. Weiskirchen R, Tacke F. Liver fibrosis: Which mechanisms matter?. Clin Liver Dis (Hoboken). 2016;8(4):94-99.

18. Farzaei MH, Zobeiri M, Parvizi F, El-Senduny FF, Marmouzi I, Coy-Barrera E, et al. Curcumin in Liver Diseases: A Systematic Review of the Cellular Mechanisms of Oxidative Stress and Clinical Perspective. Nutrients. 2018;10(7):855.
19. Zhang S, Li TS, Soyama A, Tanaka T, Yan C, Sakai Y, et al. Up-regulated extracellular matrix components and inflammatory chemokines may impair the regeneration of cholestatic liver. Sci Rep. 2016;6:26540.

20. Roderburg C, Urban GW, Bettermann K, Vucur M, Zimmermann H, Schmidt S, et al. MicroRNA profiling reveals a role for miR-29 in human and murine liver fibrosis. Hepatology. 2011;53(1):209-218.

21. Purnamaningsih SM. The serum Arginase-1 correlation to child-pugh scores in predicting the severity of cirrhosis. Bali Medical Journal. 2017;6(3):611-615.

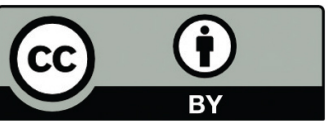

This work is licensed under a Creative Commons Attribution 